\title{
Editorial
}

\section{Photocatalysis and Photoelectrochemistry for Solar Fuels}

\author{
Zhigang Zou, ${ }^{1}$ Jinhua Ye, ${ }^{2}$ Michael R. Hoffmann, ${ }^{3}$ and Wenjun Luo ${ }^{1}$ \\ ${ }^{1}$ Department of Physics, Ecomaterials and Renewable Energy Research Center (ERERC), Nanjing University, Nanjing 210093, China \\ ${ }^{2}$ International Center for Materials Nanoarchitectonics (MANA), National Institute for Materials Science (NIMS), \\ Tsukuba 3050044, Japan \\ ${ }^{3}$ Linde-Robinson Laboratories, California Institute of Technology, Pasadena, CA 91125, USA
}

Correspondence should be addressed to Zhigang Zou; zgzou@nju.edu.cn

Received 21 October 2014; Accepted 21 October 2014; Published 1 December 2014

Copyright (c) 2014 Zhigang Zou et al. This is an open access article distributed under the Creative Commons Attribution License, which permits unrestricted use, distribution, and reproduction in any medium, provided the original work is properly cited.

The Sun generates enough energy to power the Earth. However, solar energy should be stored into chemical energy to be conveniently used due to its low energy density and discontinuous radiation. In the last several years, photocatalysis and photoelectrochemistry for solar fuels have reattracted more and more governments' and people's interest from all over the world and become a very hot topic. If we utilize the abundant solar energy to convert $\mathrm{CO}_{2}$ into hydrocarbon fuels especially, it would address the problems of global climate change and solar energy storage at the same time. Recently, different new materials and ideas have been proposed and steady scientific progress has been done. However, it is still a key challenge to explore visible-light responsive materials with high photocatalytic activities. The special issue contains eight papers, where 6 papers are related to visible-light activity and 2 papers are related to UV activity.

In a paper entitled "Development of thin film amorphous silicon tandem junction based photocathodes providing high open-circuit voltages for hydrogen production" by F. Urbain et al., they prepare the a-Si:H/a-Si:H based photocathodes, which exhibit a high photocurrent onset potential of $1.76 \mathrm{~V}$ versus the reversible hydrogen electrode (RHE) and a photocurrent of $5.3 \mathrm{~mA} / \mathrm{cm}^{2}$ at $0 \mathrm{~V}$ versus RHE. They provide an efficient and low-cost route to solar hydrogen production.

In a paper entitled "Bottom-up enhancement of $g-C_{3} N_{4}$ photocatalytic $\mathrm{H}_{2}$ evolution utilising disordering intermolecular interactions of precursor" by X. L. Wang et al., they develop a bottom-up strategy to synthesize g- $\mathrm{C}_{3} \mathrm{~N}_{4}$ photocatalysts with improved optical property and chemical structure through using disordered dicyandiamide (D-DCDA) as reaction precursor. The carbon nitride sample condensed by D-DCDA indicates higher photocatalytic activity for hydrogen evolution due to looser structure and more effective light harvesting and charge separation efficiency.

In a paper entitled "Structure, optical properties, and photocatalytic activities towards $\mathrm{H}_{2}$ generation and $\mathrm{CO}_{2}$ reduction of GaN nanowires via vapor-liquid-solid process" by $\mathrm{H}$. Pang et al., they synthesize high quality single crystalline $\mathrm{GaN}$ nanowires with large aspect ratio on n-type Si (111) substrate via the $\mathrm{Au}$-catalyzed vapor-liquid-solid process. Photocatalytic $\mathrm{H}_{2}$ evolution and $\mathrm{CO}_{2}$ reduction over the asprepared $\mathrm{GaN}$ nanowires are also investigated. Their results suggest that the GaN nanowires greatly enhanced capability compared to the $\mathrm{GaN}$ powders.

In a paper entitled "Band-gap engineering of $\mathrm{NaNbO}_{3}$ for photocatalytic $\mathrm{H}_{2}$ evolution with visible light" by $\mathrm{P}$. Li et al., they shift the photoabsorption edge of $\mathrm{NaNbO}_{3}$ to the visible-light region by $\mathrm{La}$ and Co codoping. Moreover, $\mathrm{H}_{2}$ is successfully generated over the doped $\mathrm{NaNbO}_{3}$ samples under visible-light irradiation. Density-functional theory calculations show that Co-induced impurity states are formed in the band gap of $\mathrm{NaNbO}_{3}$, which is considered to be the origin of visible-light absorption upon the doped $\mathrm{NaNbO}_{3}$ sample.

In a paper entitled "Sensitization of perovskite strontium stannate $\mathrm{SrSnO}_{3}$ towards visible-light absorption by doping" by $\mathrm{H}$. Chen and $\mathrm{N}$. Umezawa, they calculate electronic structures of $\mathrm{SrSnO}_{3}$ after $\mathrm{Cr}^{3+}, \mathrm{Fe}^{3+}$, and $\mathrm{Rh}^{3+}$ and anions $\mathrm{N}^{3-}, \mathrm{N}^{2-}$, and $\mathrm{S}^{2-}$ doping. Among all considered cation dopants, $\mathrm{Rh}^{3+}$ gives rise to the deep in gap states. The transition from $\mathrm{Rh}^{3+}$ to $\mathrm{SrSnO}_{3}$ conduction band is $0.89 \mathrm{eV}$ lower than the band gap. 
In a paper entitled "Surfactant-free synthesis of single crystalline $\mathrm{SnS}_{2}$ and effect of surface atomic structure on the photocatalytic property" by M. Li et al., they prepare sheet like tin disulfide $\mathrm{SnS}_{2}$ single crystal exposed with welldefined $\{001\}$ facets and flowerlike $\mathrm{SnS}_{2}$ mainly exposed with $\{010\}$ facets through a surfactant-free solvothermal process. The sheet like $\mathrm{SnS}_{2}$ showed a much higher photocatalytic activity in degradation of methyl orange than flowerlike $\mathrm{SnS}_{2}$. Theoretical and experimental results reveal that the band structure derived from the surface atomic structure played a more important role than the surface energy in the photocatalytic property.

In a paper entitled "Preparation of cerium modified titanium dioxide nanoparticles and investigation of their visible light photocatalytic performance" by J. Liu et al., they prepare $\mathrm{CeO}_{x} / \mathrm{TiO}_{2}$ by the hydrothermal-calcination method. The $\mathrm{Ce}$ ions have the two valence states, $\mathrm{Ce}^{3+}$ and $\mathrm{Ce}^{4+}$, which can act as the electron acceptor to improve the separation efficiency of the photogenerated electron-hole pairs.

In a paper entitled "Visible-light degradation of dyes and phenols over mesoporous titania prepared by using anthocyanin from red radish as template" by Z. Yan et al., they prepare highly crystalline mesoporous titania by using a natural pigment from red radish as template. The prepared mesoporous titania photocatalyst exhibited significant activity under visible-light irradiation for the degradation of dyes and phenols due to its red shift of band-gap-absorption onset and visible-light response as a result of the incorporation of surface carbon species.

\section{Acknowledgment}

We would like to thank all of the authors and the reviewers for their contributions to this special issue.

Zhigang Zou Jinhua Ye

Michael R. Hoffmann Wenjun Luo 

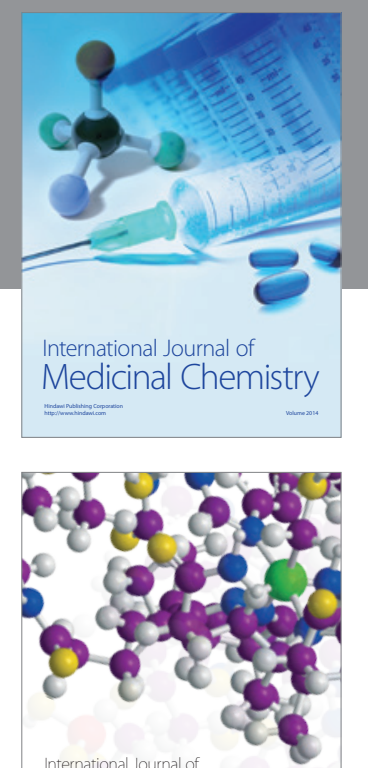

\section{Carbohydrate} Chemistry

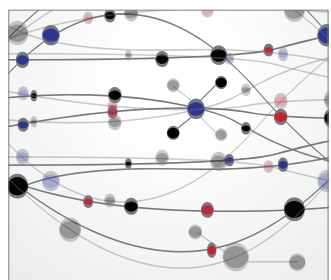

The Scientific World Journal
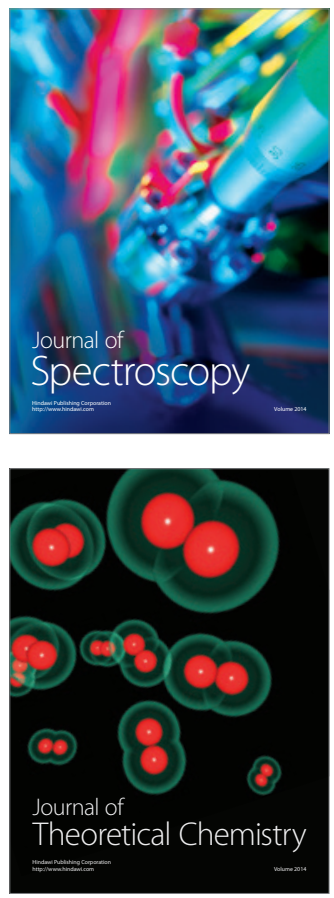
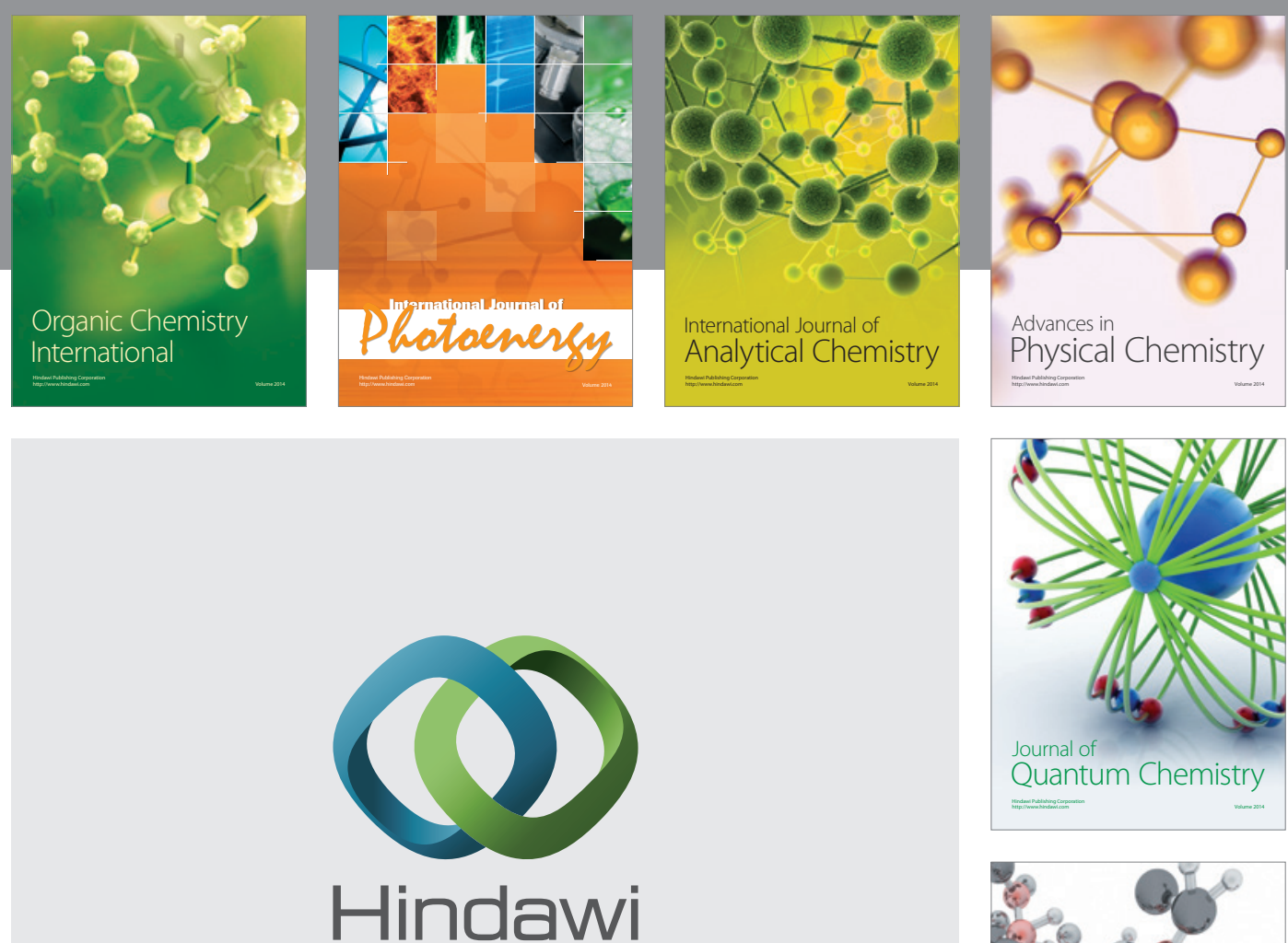

Submit your manuscripts at

http://www.hindawi.com

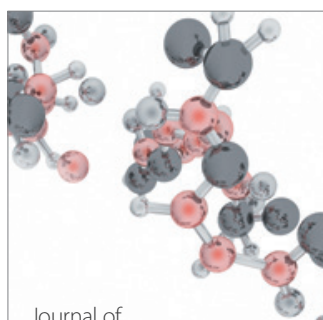

Analytical Methods

in Chemistry

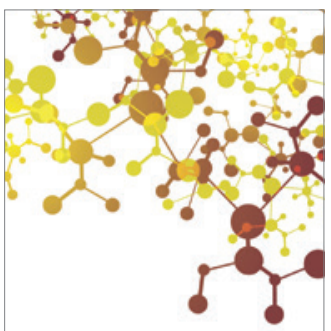

Journal of

Applied Chemistry

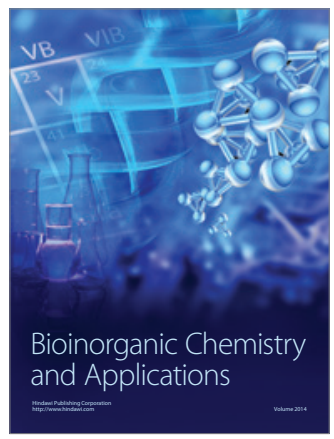

Inorganic Chemistry
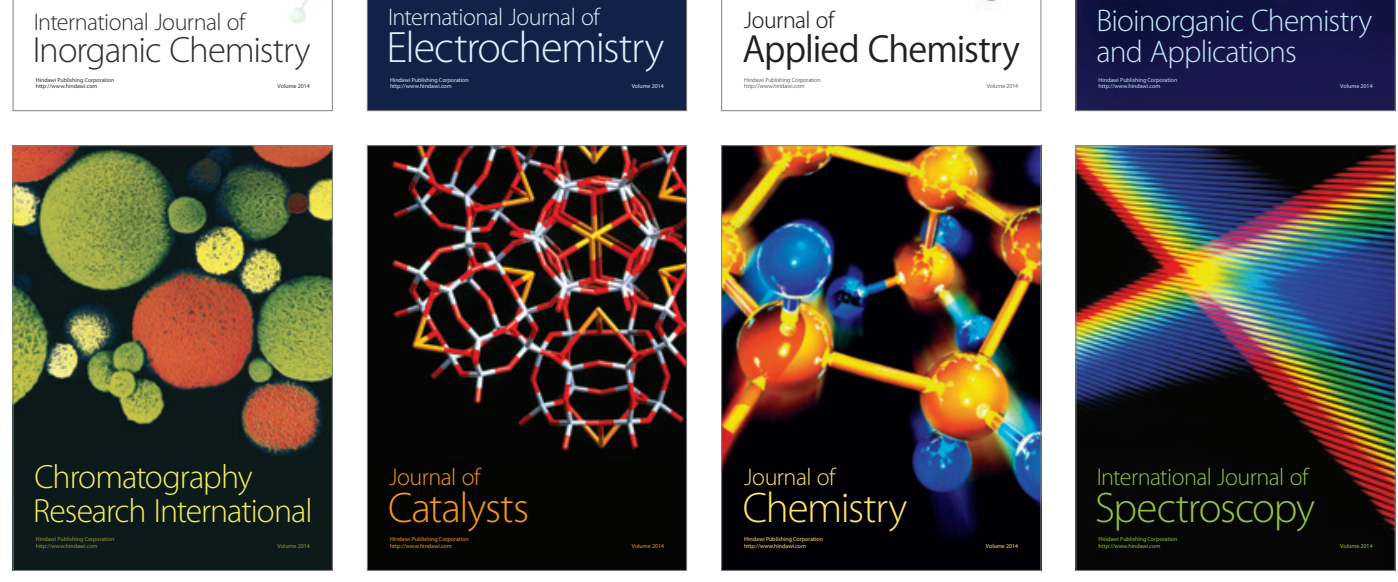